\title{
High-temperature distributed sensor system via BOTDA and multimode gold-coated fiber
}

\author{
R. Ruiz-Lombera*, I. Laarossi, J. Mirapeix, M.A. Quintela, J.M. Lopez-Higuera \\ Photonics Engineering Group, University of Cantabria, Plaza de la Ciencia s/n, Edificio \\ I+D Teleco, 39005, Santander (Spain)
}

\begin{abstract}
A high-temperature distributed sensor solution based on a Brillouin Optical Time Domain Analyzer and a multimode gold-coated fiber is presented and experimentally validated in this paper. Distributed temperature measurements up to $600^{\circ} \mathrm{C}$ will be demonstrated.
\end{abstract}

Keywords: stimulated Brillouin scattering, distributed sensing, BOTDA, gold-coated fiber, hightemperature measurements

\section{INTRODUCTION}

Distributed sensing based on optical fiber systems is nowadays a significant research line given the unique features that these solutions give rise to. The optical fiber acting as sensor can be deployed for distances of several tens of kilometers, even exceeding $100 \mathrm{~km}$ if optical amplification is considered within the system, and spatial resolutions of $1 \mathrm{~m}$ can be achieved for long distances. Stimulated Brillouin scattering (SBS) ${ }^{1}$ mainly in BOTDA (Brillouin Optical Time Domain Analysis) configurations, has been extensively explored during the last years in this regard, with continuous advances focused, not only in achieving larger measurement distances and better spatial resolutions, but also on allowing dynamic measurements $^{2}$ (e.g. vibration) or compensating some intrinsic problems such as the appearance of nonlocal effects ${ }^{3}$.

In this framework, high-temperature distributed sensing is a possible application of these systems. Hightemperature measurements might prove worthwhile in different scenarios, ranging from the oil or gas industries to different industrial sectors. In fact, some solutions based on the Raman scattering have been explored for temperatures up to about $1000^{\circ} \mathrm{C}^{4}$, and there are even some commercial solutions available. It is worth mentioning that these systems also require special fibers able to resist these hard environments. If temperatures lower than $300^{\circ} \mathrm{C}$ are to be measured, polyimide-coated fibers can be considered. However, higher temperatures will imply the necessity of deploying fibers coated with metals such as aluminum or gold ${ }^{5}$. These special fibers exhibit high attenuation due to both hydrogen ingression and microbending losses due to thermal stress. This may be a problem for Raman-based systems where the measurement of temperature is based on the detection of the intensity of the scattered signals. However, distributed systems based on the Brillouin scattering perform a frequency-based detection, thus being more suitable to scenarios where attenuation can be significant.

Brillouin systems are therefore a promising approach for high temperature distributed sensing, although only a few works have been published in this area. Wang et al. used a BOTDA system to measure temperatures up to $1000^{\circ} \mathrm{C}$, with $5 \mathrm{~m}$ spatial resolution, although the optical fiber employed in this work was not specified ${ }^{6}$. Dong et al. demonstrated 1100 and $1200^{\circ} \mathrm{C}$ distributed measurements with a BOTDA configuration using single mode fiber (SMF) and a pure-silica Photonics Crystal Fiber (PCF), respectively ${ }^{7}$.

In this paper we present a high temperature distributed sensor system (up to $600^{\circ} \mathrm{C}$ ) using a BOTDA system and a multimode gold-coated fiber, which allows its deployment in real field scenarios. This is the first time to our knowledge that this kind of fiber has been used for high temperature distributed sensing via SBS.

*ruben.ruiz@unican.es; phone (+34) 942 200877; fax (+34) 942200877; http://gif.teisa.unican.es/ 


\section{EXPERIMENTAL ISSUES}

The experimental setup depicted in Figure 1 was employed to carry out the experimental tests. The BOTDA system (Fig. 1(a)) is formed by a laser source $(\lambda=1550.92 \mathrm{~nm})$, whose output is divided by a 90/10 coupler into two branches. The upper branch generates the pump wave via a Semiconductor Optical Amplifier (SOA) (connected to a Pulse Generator (PG)) and EDFA (Erbium Doped Fiber Amplifier) and a Polarization Scrambler. The probe wave is formed in the lower branch by an Electro-Optical Modulator driven by a RF generator, followed by an isolator and the fiber under test (FUT). The system works in a balanced configuration, i.e. both Stokes and anti-Stokes signals enter the FUT and participate in their corresponding SBS processes. At the output of the system, once the SBS interaction has taken place within the FUT, the resulting optical signal is filtered by a fiber Bragg grating (FBG) and only the Stokes component is detected in the photodetector (PD), thus a gain configuration is considered. Finally, an oscilloscope is employed to acquire the BOTDA trace.

It should be noted that BOTDA systems have been traditionally implemented using monomode fibers, given their improved performance in terms of attenuation in comparison to multimode fibers. A few works have dealt with distributed measurements with multimode fibers in BOTDAs: Minardo et al. ${ }^{8}$ carried out a numerical and experimental study with graded-index multimode fibers and $\mathrm{Xu}$ et al. ${ }^{9}$ developed a bend-insensitive distributed BOTDA sensor. The fiber under test (FUT) designed for the experimental tests (Fig. 1(b)) is formed by a multimode fiber region with a main gold-coated fiber (FiberGuide AFS50/125/155G) link of $74 \mathrm{~m}$ and an additional $4 \mathrm{~m}$ gold-coated fiber coil located within a furnace chamber. A small section of a standard graded-index multimode fiber was attached to both ends of the gold-coated fiber link to make easier its handling. In addition, two more small sections of standard monomode fiber were fused at both ends to act as transition to the BOTDA system access ends.

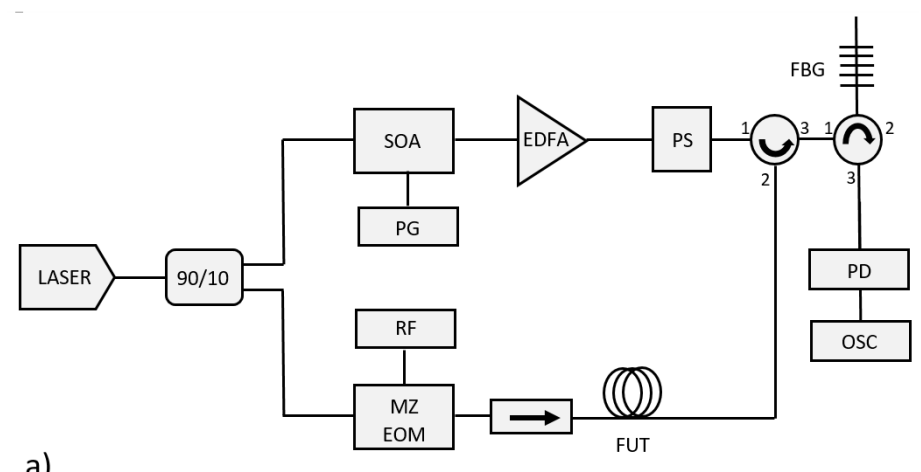

a)

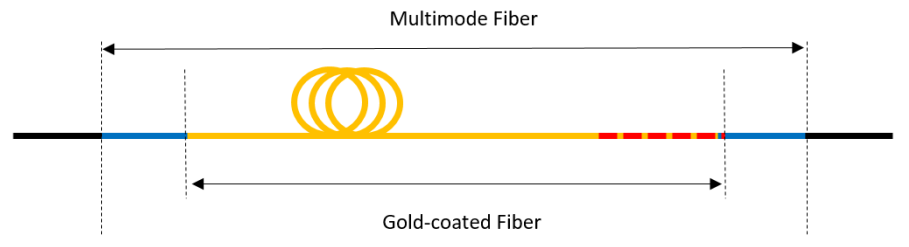

b)

Figure 1.(a) Schematic setup of the BOTDA system used in the experimental tests: Semiconductor Optical Amplifier (SOA), Pulse Generator (PG), Erbium Doped Fiber Amplifier (EDFA), Polarization Scrambler (PS), Match-Zender Electro-Optical Modulator (MZ-EOM), RF Generator (RF), Fiber Under Test (FUT), Fiber Bragg Grating (FBG), Photodetector (PD) and Oscilloscope (OSC); (b) Fiber under test employed with standard monomode fiber on both sides (black), multimode graded index fiber (blue) and gold-coated fiber of $74 \mathrm{~m}$ length (yellow) and $4 \mathrm{~m}$ length (red dashed line). 


\section{HIGH-TEMPERATURE EXPERIMENTAL TESTS}

A high-temperature furnace was used to carry out the experimental verification of the system. It is worth mentioning that a gold-coated fiber was selected due to its feasibility to be deployed in real field applications, such as industrial environments. One of the main drawbacks of this fiber is its high attenuation, mainly due to microbending losses resulting from the applied thermal stress ${ }^{5}$. In this case, an attenuation of $20 \mathrm{~dB} / \mathrm{km}$ over the deployed FUT was estimated. Figure 2 shows the results of the distributed measurements performed using a pulse width of $10 \mathrm{~ns}$ (1 m of spatial resolution). It can be observed that the differences in the Brillouin frequency shifts (BFS) associated with each temperature generated within the furnace chamber are clearly noticeable at the end of the FUT. The temperature was modified between 25 and $600^{\circ} \mathrm{C}$ with steps of $50^{\circ} \mathrm{C}$.

It can be appreciated that the temperature profiles depicted in Fig. 2 are somewhat noisy. This can be explained by the high sensitivity exhibited by the gold-coated fiber to different environmental factors, such as temperature variations or vibrations. Apart from the $4 \mathrm{~m}$ section located within the chamber furnace, the remaining $74 \mathrm{~m}$ gold-coated fiber was not protected (i.e. in a controlled environment). This explains the noise that can be appreciated in the first $74 \mathrm{~m}$ section of the distributed temperature profiles. The gold-coated fiber does not exhibit BFS hopping ${ }^{7}$ given that it was previously annealed.

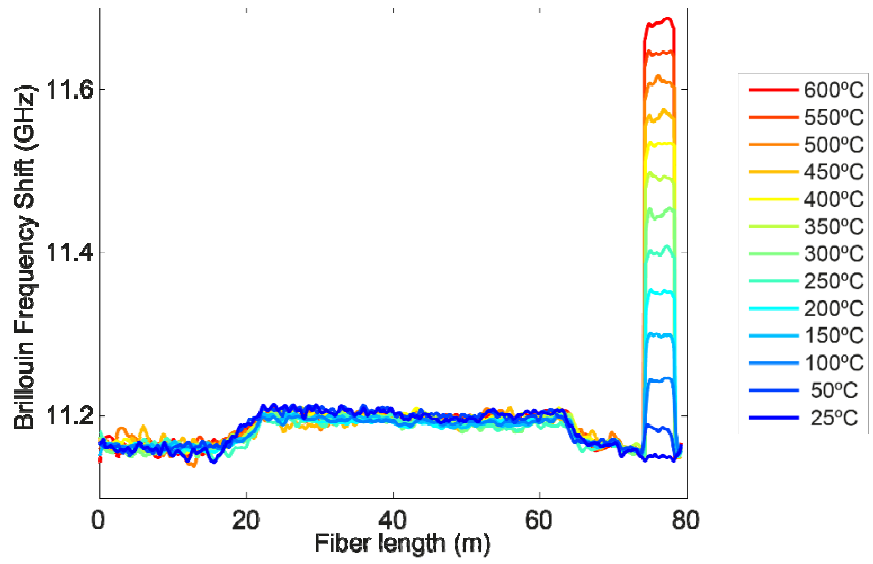

Figure 2. Experimental validation of the proposed system. Response of the BOTDA system (Brillouin frequency shift) for temperatures from $25^{\circ} \mathrm{C}$ to $600^{\circ} \mathrm{C}$.

Figure 3 presents the Brillouin frequency shift (BFS) for each temperature considered in the hotspot section at the end of the FUT.

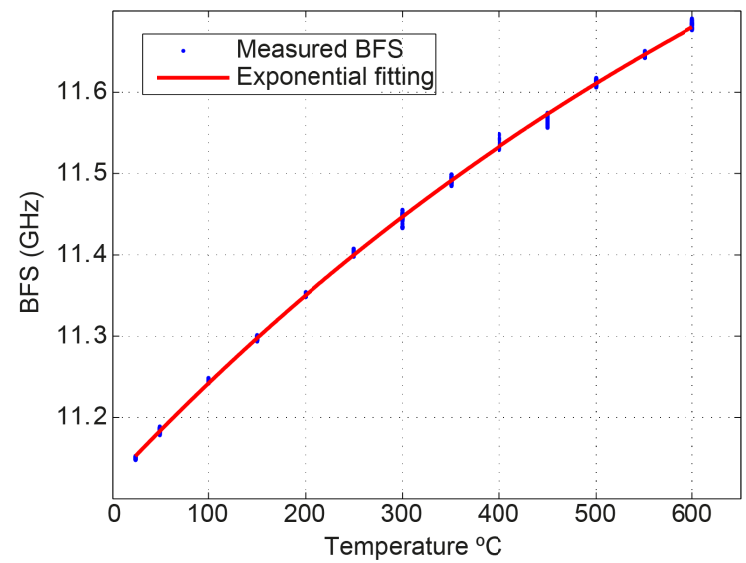

Figure 3. Brillouin frequency shift for each considered temperature at the hotspot section and the exponential fitting performed. 
The data of Fig. 3 have been fitted to an exponential curve using the least squares method through the Levenberg-Marquardt algorithm, obtaining the following expression:

$$
B F S=-1147 e^{-0.0011 T}+12268.1
$$

where the BFS is expressed in $\mathrm{MHz}$ and $T$ is the temperature in Celsius. Using this exponential curve the value of $R^{2}$ is 0.9994 and the mean error between the measured data and the real temperature is $\pm 3.68{ }^{\circ} \mathrm{C}$.

\section{CONCLUSIONS}

A high-temperature distributed sensing system based on a BOTDA implementation and a multimode gold-coated fiber has been presented in this work. This is the first time to our knowledge that a multimode gold-coated fiber has been used in a Brillouin-based distributed system for high-temperature measurements. Although a few previous works have dealt with high-temperature sensing via BOTDAs, the gold-coated fiber ensures a feasible fiber deployment for temperatures up to $600^{\circ} \mathrm{C}$. Experimental tests carried out with a furnace have shown the validity of this proposal, where a spatial resolution of $1 \mathrm{~m}$ and a total effective sensing fiber length of $78 \mathrm{~m}$ have been considered. The resulting temperature accuracy is $\pm 3.6^{\circ} \mathrm{C}$. Future works will explore longer sensing fiber lengths, an improvement of the system performance in terms of the resulting signal-to-noise ratio and field tests in a real industrial scenario.

\section{ACKNOWLEDGEMENTS}

This work has been supported by the Universidad de Cantabria and by the Spanish Ministerio de Ciencia e Innovación through project TEC2013-47264-C2-1-R.

\section{REFERENCES}

[1] X. Angulo-Vinuesa, S. Martin-Lopez, J. Nuño et al., "Raman-assisted Brillouin distributed temperature sensor over $100 \mathrm{~km}$ featuring $2 \mathrm{~m}$ resolution and $1.2 \mathrm{C}$ uncertainty," Journal of Lightwave Technology, 30(8), 1060-1065 (2012).

[2] J. Urricelqui, A. Zornoza, M. Sagues et al., "Dynamic BOTDA measurements based on Brillouin phase-shift and RF demodulation," Optics express, 20(24), 26942-26949 (2012).

[3] R. Ruiz-Lombera, J. Urricelqui, M. Sagues et al., "Overcoming Nonlocal Effects and Brillouin Threshold Limitations in Brillouin Optical Time-Domain Sensors," Photonics Journal, IEEE, 7(6), 1-9 (2015).

[4] H. Fujimori, M. Kakihana, K. Ioku et al., "Advantage of anti-Stokes Raman scattering for hightemperature measurements," Applied Physics Letters, 79(7), 937-939 (2001).

[5] T. Reinsch, and J. Henninges, "Temperature-dependent characterization of optical fibres for distributed temperature sensing in hot geothermal wells," Measurement Science and Technology, 21(9), 094022 (2010).

[6] J. Wang, D. Hu, D. Y. Wang et al., "Fully-distributed fiber-optic high temperature sensing based on stimulated Brillouin scattering." 87220E-87220E-7.

[7] Y. Dong, P. Xu, C. Fu et al., " $1200^{\circ}$ C high-temperature distributed Brillouin optical fiber sensing based on photonics crystal fiber." 963485-963485-4.

[8] A. Minardo, R. Bernini, and L. Zeni, "Experimental and numerical study on stimulated Brillouin scattering in a graded-index multimode fiber," Optics express, 22(14), 17480-17489 (2014).

[9] P. Xu, Y. Dong, J. Zhang et al., "Bend-insensitive distributed sensing in singlemode-multimodesinglemode optical fiber structure by using Brillouin optical time-domain analysis," Optics express, 23(17), 22714-22722 (2015). 